\title{
The RMS survey: Massive young stars throughout the galaxy
}

\author{
M. G. Hoare ${ }^{1}$, S. L. Lumsden ${ }^{1}$, R. D. Oudmaijer ${ }^{1}$, \\ J. S. Urquhart ${ }^{1}$, A. L. Busfield ${ }^{1}$, T. L. Sheret ${ }^{1}$, A. J. Clarke ${ }^{1}$, \\ T. J. T. Moore ${ }^{2}$, J. Allsopp ${ }^{2}$, M. G. Burton ${ }^{3}$, C. R. Purcell ${ }^{3}$, \\ Z. Jiang ${ }^{4}$ and M. Wang ${ }^{4}$ \\ ${ }^{1}$ School of Physics and Astronomy, University of Leeds, LS2 9JT, UK \\ email: mgh@ast.leeds.ac.uk \\ ${ }^{2}$ Institute for Astrophysical Research, Liverpool John Moores University \\ Twelve Quays House, Egerton Wharf, Birkenhead, UK \\ ${ }^{3}$ School of Physics, University of New South Wales, Sydney, Australia \\ ${ }^{4}$ Purple Mountain Observatory, Nanjing, P. R. China
}

\begin{abstract}
We describe a programme that aims to increase the known sample of massive young stellar objects (MYSOs) by an order of magnitude. About 2000 candidates colour-selected from the MSX survey are being followed up at radio, $\mathrm{mm}$ and IR wavelengths to identify genuine MYSOs from the UCHII regions and other contaminants. Results so far indicate that the strategy does indeed deliver a significant fraction of luminous YSOs that will provide the basis for future galaxy-wide systematic studies.
\end{abstract}

Keywords. infrared: stars, radio continuum: ISM, radio lines: ISM, stars: formation, H II regions

\section{Introduction}

Massive stars are by their very nature rare and during their short-lived early phases this is amplified further. There are likely to be only a handful of the most luminous $\left(\mathrm{L}>10^{5} \mathrm{~L}_{\odot}\right)$ examples present in the entire galaxy before they reach the stage where they they ionise their surroundings as ultra-compact H II regions. If we want to study how the physical processes that drive star formation change with luminosity then a large, well-selected sample is required. Such a sample should include sufficient numbers of objects in each luminosity bin to account for the suspected stochastic nature of the star formation process. To avoid biasing any studies the samples must be selected in a well-defined way.

Unbiased searches have to begin in the IR where most of the bolometric luminosity emerges after re-processing by dust. The only problem then is that several other types of source have very similar IR colours such as UCHIIs, young planetary nebulae (PN) and dusty evolved stars (i.e. any centrally-heated, optically thick, dust cloud), and a large amount of follow-up work is needed to distinguish between them. Several attempts at systematic searches for young massive stars have been made using the IRAS PSC. Campbell, Persson \& Matthews (1989) and Chan, Henning \& Schreyer (1996) made little or no attempt to identify possible contaminating sources, whilst Molinari et al. (1998) and Sridharan et al. (2002) constructed samples that avoided H II regions detected in singledish radio surveys. This did not preclude contamination by weaker and more compact $\mathrm{H}$ II regions as later higher resolution radio studies often showed. It also has the affect of deliberately biasing the sample towards more isolated star forming regions, which can be useful for detailed individual studies but not for accessing the full picture. However, 
the main problem with any IRAS-based search is its poor spatial resolution. In densely clustered environments and close to the galactic mid-plane, IRAS was invariably confused and only produced upper limits. These are precisely the locations that a systematic search needs to include as the scale height of massive stars is very small ( $\left.\sim 30^{\prime}\right)$ (Reed 2000).

Higher resolution IR surveys are the key to getting a truly representative sample of massive young stars. The $18^{\prime \prime}$ resolution survey of the whole Galactic plane $\left(|b|<5^{\circ}\right)$ at $8,12,14$ and $21 \mu \mathrm{m}$ carried out by the MSX satellite (Price et al. 2001) provides just such an opportunity. Although its sensitivity is similar to IRAS, its beam size is 50 times smaller at 12 and $25 \mu \mathrm{m}$. The ISOGAL mid-IR survey went deeper at higher resolution, but only covered a small area (Omont 2003). Recently, the Spitzer GLIMPSE Legacy programme at somewhat shorter wavelengths has covered large parts of the inner galaxy at unprecedented resolution and sensitivity. However, the MSX survey is best suited to the deliver of a sample of the most luminous objects right across the whole galaxy.

Of course, demanding that a source is detected in the mid-IR in itself introduces a bias. Only sources that are relatively well advanced on their early evolution will have emerged sufficiently from their natal cloud to shine through in the mid-IR. We refer to such luminous $\left(\mathrm{L}>10^{4} \mathrm{~L}_{\odot}\right)$, embedded mid-IR point sources that have yet to start ionising their surrounding ISM as massive young stellar objects (MYSOs). Such a tradition spans back at least to Henning et al. (1984) who described their other typical characteristics of associated bipolar molecular flows and maser emission. They also referred to them as BN objects after the prototype in Orion which now, like many other prototypes, appears to be somewhat atypical (Tan, Rodriguez this volume). This mid-IR bright MYSO stage would appear to normally follow the so-called 'hot molecular core' stage, which is usually invisible or difficult to detect in the mid-IR (de Buizer et al. 2002). These are marked out by the liberation of certain molecular species from ices as the core heats up. Methanol is one such species and methanol masers have also been used to trace such early phases. However, there is no clear-cut distinction since some mid-IR bright MYSOs also have associated methanol maser emission, whilst many others do not (Beuther et al. 2002). A fully representative sample of the earlier hot and cold core stages selected from their bolometric flux will have to wait for the much longer wavelength higher resolution plane surveys from HERSCHEL (Molinari \& Swineyard 2001) and SCUBA2.

The MYSOs are almost certainly already burning hydrogen in their cores as the KelvinHelmholtz contraction timescale is so short for these massive objects (Behrend \& Maeder 1996). However, it is also likely that they are still accreting. Examples that are sufficiently bright in the near-IR often exhibit the $\mathrm{CO}$ bandhead in emission with line profiles that have been interpreted as typical double-peaked accretion disc profiles (Chandler et al. 1993; Blum, this volume). Ongoing accretion onto these stars will have the effect of significantly increasing their radius (Palla, this volume). This will also cause the stellar effective temperature and the characteristic accretion disc temperature to be lower. This could explain why these luminous objects are not already ionising the surrounding cloud they are simply not hot enough. It has been noted for many years that the emission line spectra share characteristics with the low surface gravity evolved hypergiant stars of similar luminosity (e.g. Simon \& Casar 1984; Bunn, Hoare \& Drew 1995). In the picture outlined above accretion via a disc is naturally accounted for whereas the quenching of the H II region by infalling material (Walmsley 1995) requires high infall rates in all directions. Such an explanation would require the accretion rate to fall below some critical level before ionisation of the surroundings can begin and thus would mark out the beginning of the UCHII phase as the end of the accretion phase.

The list of 30 or so well-studied examples of MYSOs that Henning et al. (1984) compiled has about doubled over the years, but not in any systematic way. It is our aim to 
deliver a bona fide sample that is an order of magnitude larger, covers all star-forming environments in the galaxy, and can be used for statistical studies and selection of subsamples for specific follow-up investigations.

\section{Object Selection}

We have investigated the use of colour-selection to separate different populations in the combined MSX and 2MASS data (Lumsden et al. 2002). A detection in 2MASS is not required, these data are merely used to eliminate sources that are too blue. Using version 2 of the MSX PSC and eliminating sources that do not appear point-like in the images we obtain a sample of nearly 2000 sources with $21 \mu \mathrm{m}$ flux above $2.5 \mathrm{Jy}$ and colours consistent with known MYSOs - see the triangles in Figure 1. The dots in the background are a representative sample of all MSX sources, which clearly show the location of bright, relatively unreddened, stars. $70 \%$ of these stars are eliminated by our colour-colour cuts. Also over-plotted are examples of known ultra-compact H II regions (squares), PN (*) and $\mathrm{OH} / \mathrm{IR}$ stars $(+)$. Only about $25 \%$ of these red MSX sources passing our colour cuts have un-confused IRAS data and the vast majority are unknown in the literature.
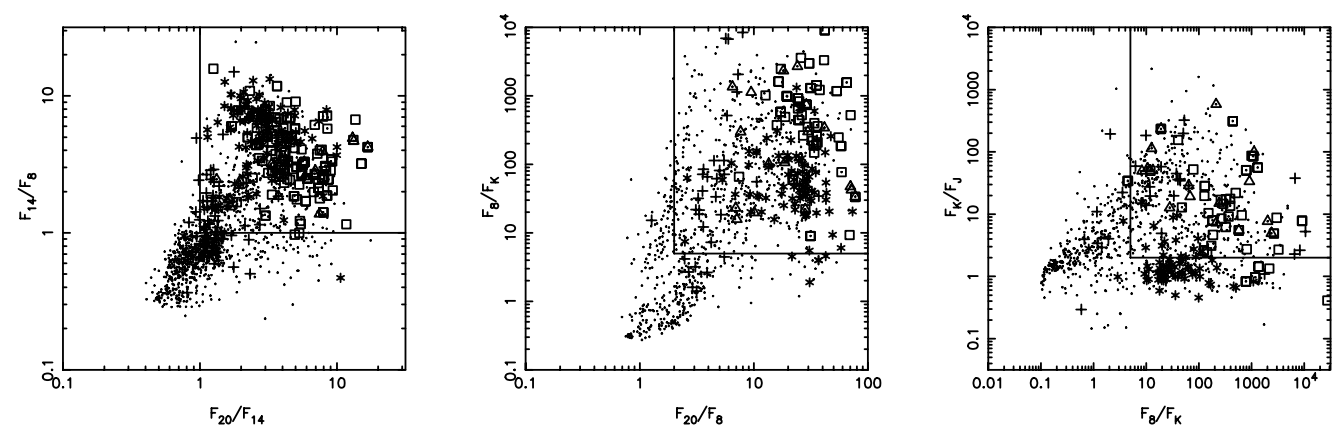

Figure 1. Colour-colour plots using MSX and 2MASS data (see text for symbols).

\section{Multi-Wavelength Identification Campaign}

The core of the red MSX source, or RMS, survey is a multi-wavelength campaign to distinguish genuine MYSOs from the other confusing sources that have the same colours. Since UCHIIs are likely to be the main contaminant radio continuum observations are essential. We have recently completed $5 \mathrm{GHz}$ observations to $1 \mathrm{mJy}$ sensitivity at $1^{\prime \prime}$ resolution of all the candidates using the VLA and ATCA. This reveals all bright UCHIIs (and compact PN) near the MSX source position. High spatial resolution is required due to the frequent crowding of young massive stars at different stages and the relatively poor accuracy of the MSX coordinates $\left(\approx 2^{\prime \prime}\right)$. We do not want to bias our sample by eliminating suspected UCHIIs when in fact the more luminous IR source was a nearby MYSO that does not show up in the radio. This is accomplished through higher resolution mid-IR imaging where both the MYSOs and UCHIIs are prominent sources. Ground-based $10 \mu \mathrm{m}$ imaging at sub-arcsecond resolution has been carried out at UKIRT and the ESO 3.6m. Within the GLIMPSE survey area the SPITZER data are being used for this purpose. Figure 2 shows an example of our ground-based imaging whereby a point source appears in close proximity to an extended source, which is likely to be $\mathrm{Ly}-\alpha$ heated dust in a compact H II region. This source was not observed by us in the radio since it is also in the Sridharan et al. (2002) sample and Beuther et al. (2002) present VLA data that 


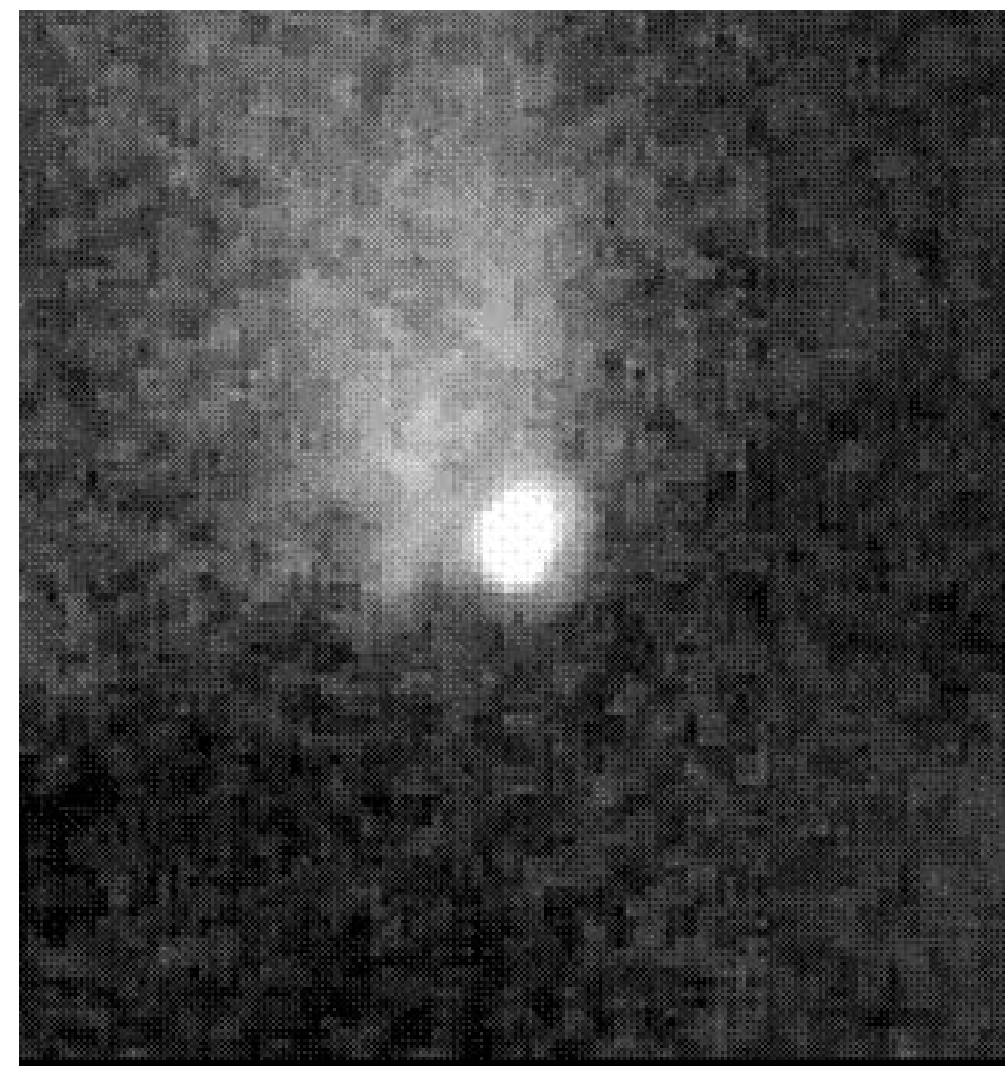

Figure 2. $10 \mu \mathrm{m}$ image of an RMS source (G109.0982-00.3454, IRAS22570+5912) showing a point source ahead of a cometary extended source, most likely a compact H II region. The image was taken at UKIRT with MICHELLE and is $20^{\prime \prime}$ square.

show the presence of a radio source offset from the MSX position by a few arcseconds consistent with the above interpretation. Point sources remain as candidate MYSOs, whilst extended sources are eliminated. The higher resolution mid-IR data also allows a search for multiplicity within the MSX beam and as a check on whether any point source actually dominates the MSX flux. Current indications are that a single point source that accounts for most of the MSX flux is the norm.

With photo-ionized and any other diffuse sources removed the remaining contaminants are nearby low-luminosity YSOs and evolved stars. To identify these we are about to complete ${ }^{13} \mathrm{CO}$ observations of the entire sample. The observations of the $1-0$ line at Mopra, Onsala and PMO and 3-2 line at the JCMT allow the kinematic distance to be derived. Inside the solar circle the near/far ambiguity has to be solved and for this we are using the H I self-absorption method (Jackson et al 2002). We have so far used the SGPS H I survey data (McClure-Griffiths et al. 2001) in the fourth quadrant (Busfield et al. 2005) and will utilise the rest of the IGPS (www.ras.calgary.ca/IGPS) for the remainder. For objects with unambiguous distances and good IRAS data the luminosity can then be derived. Figure 3 shows the distribution of luminosities we have obtained so far for sources in the outer galaxy. It shows that about a third of the sources have a luminosity $>10^{4} \mathrm{~L}_{\odot}$, and that we are also finding many intermediate luminosity sources that are likely to be embedded precursors to the optically visible Herbig AeBe stars. 


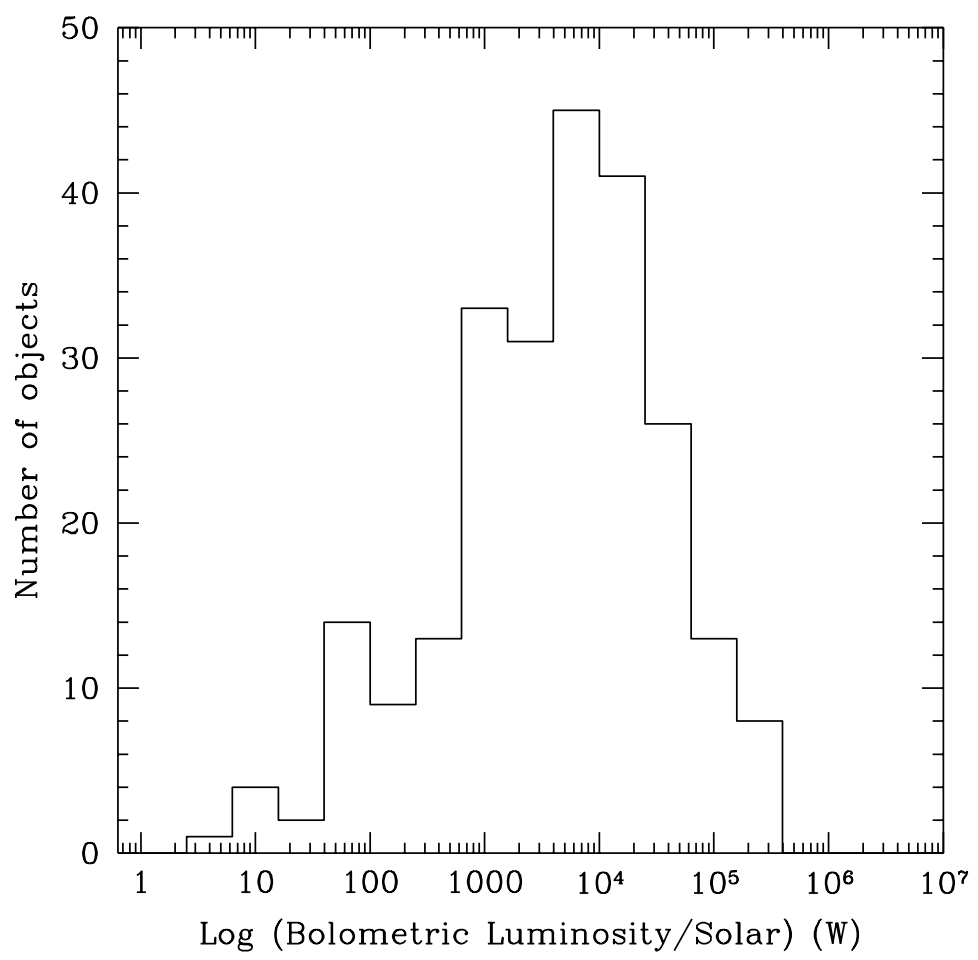

Figure 3. Histogram showing the distribution of known luminosities of sources in the RMS survey so far. These are outer galaxy objects that have no distance ambiguity and have good IRAS data. Kinematic distances are from our ${ }^{13} \mathrm{CO}$ observations.

Evolved stars do not normally have such strong CO lines as star forming regions and this can help to identify them. They also tend to appear isolated in 2MASS, MSX and GLIMPSE images. In the crowded inner galaxy there will be many instances where evolved stars are seen along the line of sight towards molecular clouds. To identify these more pathological cases, near-IR spectra provide the final confirmation. Cool star spectral features are easily distinguishable from the rather featureless spectra of MYSOs. The 150 or so spectra obtained so far from UKIRT confirm that our final sample are mostly genuine MYSOs.

\section{Conclusions}

We are in the process of delivering a sample of MYSOs that will be large and sufficiently free from biases to address many questions in massive star formation. For example, the luminosity function, lifetime and clustering will be studied as functions of location in the Galaxy. Possible triggering scenarios can be investigated by proximity to older star clusters, H II regions,etc. Sub-samples will be selected from the RMS survey to address particular questions as functions of luminosity and location. We will make the sample available at www.ast.leeds.ac.uk/RMS.

\section{References}

Aspin, C., et al. 1994, A\&A, 292, L9

Behrend R. \& Maeder A. 2001, A\&A, 373, 190 
Beuther, H., Walsh, A., Schilke, P., Sridharan, T. K., Menten, K. M. \& Wyrowski, F. 2002, $A \mathscr{E} A, 390,289$

Bunn J.C., Hoare M.G. \& Drew J.E., 1995, MNRAS, 272, 346

Busfield A. L., Hoare, M. G., Lumsden S. L., Oudmaijer R. D., Moore T. J. T., Sheret T. K. \& Purcell C. R. 2005, MNRAS, submitted

Campbell B., Persson S.E. \& Matthews K. 1989, AJ, 98, 643

Chan S., Henning Th. \& Schreyer K. 1996, A\&AS, 115, 285

Chandler C. J., Carlstrom J. E., Scoville N. Z., Dent W. R. F. \& Geballe T. R. 1993, ApJ, 412, L71

De Buizer, James M., et al. 2002, ApJ, 564, L101

Henning Th., Friedmann C., Gürtler J. \& Dorschner J., 1984, Astron. Nactr., 305, 67

Jackson, J. M., Bania, T. M., Simon, R., Kolpak, M., Clemens, D. P. \& Heyer, M. 2002, ApJ, $566, \mathrm{~L} 81$

Lumsden S. L., Hoare, M. G., Oudmaijer R. D. \& Richards D. 2002, MNRAS, 336, 621

McClure-Griffiths, N. M., et al. 2001, ApJ, 551, 394

Molinari S. \& Swineyard, B. M. 2001, in: Pilbratt G. L., Chernicaro, J., Heras, A. M., Prusti, T., Harris, R. The Promise of the Herschel Space Observatory, ESA-SP 460, 207

Molinari, S., Brand, J., Cesaroni, R., Palla, F. \& Palumbo, G. G. C. 1998, A\& $\&$, 336, 339

Omont, A., et al. 2003, A\&A, 403, 975

Price S.D., Egan M.P., Carey S.J., Mizuno D.R. \& Kuchar T.A. 2001, AJ, 121, 2819

Reed B.C. 2000, $A J, 120,314$

Simon M., Cassar L. 1984, ApJ, 283, 179

Sridharan T.K., Beuther H., Schilke P., Menten K.M. \& Wyrowski F. 2002, ApJ, 566, 931

Walmsley C.M. 1995, Rev. Mex. Ast. Ap. Conf. Ser., 1, 137 